\title{
INFLUENCE OF FOLIAR APPLICATION OF SALICYLIC ACID ON GROWTH AND FLOWERING OF CALENDULA OFFICINALIS L. UNDER LEVELS OF SALINITY
}

\author{
M. E. Abd El Gayed \\ Botanical Gardens Res. Depart., Hort. Res. Instit. ARC. Giza, Egypt \\ Received: Oct. 13,2020 \\ Accepted: Oct. 21,2020
}

\begin{abstract}
Global climatic changes limited the fresh water supply, which led to increasing the need for irrigation water. For this reason, the main objective of this study was to investigate the influence of diluted seawater irrigation $(0,10,20$ and $30 \%$ seawater) and trying to counteract the adverse effect of seawater salinity by spraying with salicylic acid $(S A)(0,150$, and $300 \mathrm{ppm})$. Additionally, during the two successive seasons of 2017 and 2108 seasons an experimental pots research in Sakha horticultural research station at Kafr El-Sheik Governorate, Egypt, was performed to study the effects of irrigation with diluted seawater combined with foliar application of salicylic acid at on growth, flowering and survival percentage parameters, along with the chemical composition of Calendula officinalis $L$. plants. The results exposed that irrigation with high levels of diluted seawater at 20 and $30 \%$ decreased growth, root and flowering parameters, total green color, carotene contents in flowers and leaf $\mathrm{N}, \mathrm{P}, \mathrm{K}, \mathrm{Ca}$ contents and survival \% meanwhile, $\mathrm{Na}$, $\mathrm{Cl}, \mathrm{Na} / \mathrm{K}$ ratio and proline were increased. All tested foliar applications of SA especially at $300 \mathrm{ppm}$ increased all vegetative growth, roots and flowering traits. The most effective treatments which enhance growth, flowering parameters, mineral contents, and survival\% were found to be application of 0 or $10 \%$ seawater in combination with spraying SA at 150 and $300 \mathrm{ppm}$. Thus, it could be recommended to irrigate Calendula plant with diluted seawater at $10 \%$ with spraying salicylic acid at $300 \mathrm{ppm}$ to give the best results in terms of growth, flowering and survival \%.
\end{abstract}

Key words: Calendula officinalis L., seawater, salicylic acid, growth, flowering parameters

\section{INTRODUCTION}

Calendula officinalis L. (Family, Asteraceae) known as Calendula or pot marigold is an annual specie widely used around the world as a medicinal, gardens and landscape plant. It grows in sun or partial shade and is easy to grow requiring little cultivation (Dole and Wilkins, 2004). There are numerous varieties of this species, differing primarily in flower shape and color (Hamburger et al., 2003).

In abiotic stresses, drought and salinity are two environmental factors, which are responsible for the huge loss in agricultural demand and productivity all over the world (Gul and Ahmad, 2004).
Water scarcities were expected to lead to loss of crop production globally up to $\mathbf{3 0}$ $\%$ by 2025 year compared to the current yield. Salinity of agricultural soil is becoming a serious issue due to increasing shortage of fresh water and subsequent necessity to utilize saline water or low-quality water for irrigation (Semiz and Suarez, 2015). Therefore, at the current situation of intention for improving agricultural production under limited water resources, it is required to grow crops that are capable of tolerating drought and water stress (Yao et al., 2016). It would be the most economical approach to improve productivity based on limited water resources. Agricultural 
production consumes more fresh water than any other human activity. To cope with the scarcity of fresh water for the sustainable development of agriculture, there is increasing awareness among agricultural scientists and planners in the utilization of seawater (at least diluted) for irrigation of crops (Jin et al., 1999 and Liu et al., 2003). To increase the sustainable agriculture as much as the use of saline water, there are two traits have to be considered: select the plants tolerant to salinity and treating the plants to enhance their cope to the salinity (Cassaniti et al., 2012). At the latest two decades, the global climate relatively had changed which already affected the environment in many ways. The most impacts of global climate change are climate drought and a shortage of irrigation water, especially in arid and semiarid regions. Likely, Egypt will be suffering the plight of water shortage in next decades. Therefore, the search for other sources of irrigation water should be a priority. One of another sources of water irrigation is the use of diluted seawater to compensate for the shortage of irrigation water. The high concentration of ionic elements in seawater is the main restricting factor in the utilization of seawater for irrigation ( Xiao-Hua et al., 2009). There are several disadvantages when irrigation with saline water induced abiotic stress and toxic effects on plants which lead to gradual decline in photosynthesis and respiration rates and deterioration in, proteins and nucleic acids ((Munns et al., 1995). Salicylic acid (2-hydroxy benzoic acid) is an endogenous growth regulator of the phenol nature, which participates in the regulation of physiological processes in plants. It plays an important role in the plant response to adverse environmental conditions such as salinity (Baby et al., 2010). Salicylic acid biosynthetic pathway in plants has two distinct pathways, the isochorismate (IC) pathway and the phenylalanine ammonialyase (PAL) pathway. Moreover, salicylic acid plays exclusive role in plant growth, thermo genesis, flower induction and uptake of ions and has diverse effects on tolerance to abiotic stress (Van Breusegem et al., 2001). It affects ethylene biosynthesis, stomatal movement and reverses the effects of $A B A$ on leaf abscission (Yusuf et al., 2013). Therefore, the objective of this study was to evaluate the effects of diluted seawater irrigation and salicylic acid treatments on growth and flowering parameters of calendula plant.

\section{MATERIALS AND METHODS}

A pot experiment was carried out during the two successive seasons of 2017/2018 and 2018/2019. Trials took place in Sakha horticultural research station at Kafr El-Sheikh Governorate, Egypt to investigate the influence of diluted seawater irrigation $(0,10,20$, and $30 \%)$ on growth, flowering and chemical composition of pot marigold plant (Calendula officinalis L.). Furthermore, trying to counteract the adverse effect of seawater salinity by spraying with salicylic acid at (0,150 and $300 \mathrm{ppm})$. In the Sakha Horticultural Research Station, local seeds of pot marigold (Calendula officinalis L.) were sown in polyethylene plug trays filled with a mixture of peat moss and vermiculite $(2: 1 \mathrm{v} I \mathrm{v})$ on October $1^{\text {St }}$ in both growing seasons. The seedlings were transplanted in the pots after 30 days from sowing; the seedlings were planted individually in black plastic pots $(20 \mathrm{~cm}$ diameter) packed with $7 \mathrm{~kg}$ sandy clay soil (2 clay: 1 sand v/v) and it was stayed in open field. Soil samples were taken before the establishment of the study for of the chemical properties (Table, 1) according to Jackson (1967).

Seawater for irrigation was taken from the sea in Baltim town. Its salinity was approximately $42500 \mathrm{ppm}$ in the first and second seasons, respectively. Before irrigation, seawater was diluted with tap 
water in a plastic tank to the needed dilution $0,10,20$ and $30 \%$ seawater. The diluted seawater at various concentrations was used for irrigation after transplanting 20 days through the course of the experiment. The chemical properties of seawater and diluted seawater used for irrigation are shown in Tables (2 and 3).

Three concentrations of salicylic acid were used 0,150 and $300 \mathrm{ppm}$ as foliar spraying. Salicylic acid was dissolved in an absolute ethanol and then added to water (ethanol: water, 1: $1000 \mathrm{v} / \mathrm{v}$ ) as explained by Williams et al., (2003). Spraying of SA was done after approximately one month from transplanting (November 1 $\underline{\text { st }}$ ) and was repeated every 15 days for 3 times. Foliar sprayings were applied using a hand-held sprayer. To prevent interferences with different moisture levels, the same quantity of distilled water was sprayed to the control plants at the same time.

The experiment layout was set in a completely randomized design in factorial with two factors (4 seawater irrigation levels $\times 3$ salicylic acid concentrations). Each treatment was replicated three times with nine seedlings for each replicate (3 replicates $\mathrm{x} 9$ seedlings).

Table (1): Physical and chemical properties of the used soil

\begin{tabular}{|c|c|c|c|c|c|c|c|c|c|}
\hline \multirow[t]{2}{*}{$\begin{array}{c}E c \\
\left(\mathrm{dSm}^{-1}\right)\end{array}$} & \multirow[t]{2}{*}{ O.M (\%) } & \multirow[t]{2}{*}{$\mathrm{pH}$} & \multicolumn{4}{|c|}{ Soluble cations (meq/l) } & \multicolumn{3}{|c|}{$\begin{array}{l}\text { Soluble anions } \\
\text { (meq/l) }\end{array}$} \\
\hline & & & $\mathrm{Ca}^{++}$ & $\mathbf{M g}^{++}$ & $\mathrm{K}^{+}$ & $\mathrm{Na}^{+}$ & $\mathrm{So}_{4}^{--}$ & $\mathrm{HCO}_{3}^{-}$ & $\mathrm{Cl}^{-}$ \\
\hline 1.36 & 1.24 & 7.79 & 3.5 & 2.98 & 0.18 & 6.93 & 4.03 & 7.90 & 1.66 \\
\hline \multicolumn{3}{|c|}{ Particle size divisions (\%) } & Textural & \multicolumn{6}{|c|}{ Available nutrients (ppm) } \\
\hline Sand & Silt & Clay & & $\mathbf{N}$ & & $\mathbf{P}$ & & K & \\
\hline 36.37 & 21.50 & 42.13 & Clayey Sand & 233.75 & & L.31 & & 440.15 & \\
\hline
\end{tabular}

Table (2): Chemical analysis of seawater (mean of two seasons)

\begin{tabular}{lccccccccc}
\hline $\begin{array}{c}\text { lons } \\
\text { Sample }\end{array}$ & $\begin{array}{c}\mathrm{N} \\
\%\end{array}$ & $\begin{array}{c}\mathrm{P} \\
\mathrm{ppm}\end{array}$ & $\begin{array}{c}\mathrm{K} \\
\mathrm{ppm}\end{array}$ & $\begin{array}{c}\mathrm{Ca} \\
\mathrm{ppm}\end{array}$ & $\begin{array}{c}\mathrm{Mg} \\
\mathrm{ppm}\end{array}$ & $\begin{array}{c}\mathrm{Na} \\
\mathrm{ppm}\end{array}$ & $\begin{array}{c}\mathrm{Cl} \\
\mathrm{Meq} / \mathrm{l}\end{array}$ & $\mathrm{pH}$ & $\begin{array}{c}\mathrm{EC} \\
\mathrm{dsm}^{1}\end{array}$ \\
\hline Seawater & 1.45 & 116.14 & 252 & 32 & 175 & 13805 & 1.6 & 8.33 & 57.4
\end{tabular}

Table (3): $\mathrm{pH}$ and EC values of the various diluted seawater treatments at the starting of the experiment

\begin{tabular}{lcccc}
\hline Seawater (\%) & \multicolumn{2}{c}{$\mathrm{pH}$} & \multicolumn{2}{c}{$\mathrm{EC}\left(\mathrm{dsm}^{-1}\right)$} \\
\cline { 2 - 5 } & 2017 & 2018 & 2017 & 2018 \\
\hline 0 & 8.5 & 8.31 & 0.49 & 0.46 \\
\hline 10 & 7.81 & 8.11 & 5.10 & 7.77 \\
\hline 20 & 7.99 & 8.12 & 8.56 & 8.88 \\
\hline 30 & 7.78 & 8.00 & 17.32 & 18.60
\end{tabular}


Data recorded:

Vegetative and root growth measurements and survival \% :

Vegetative and root growth parameters were recorded after 90 days from transplanting and included: plant height (cm), number of shoots plant $^{-1}$ number of leaves plant ${ }^{-1}$, total leaf area plant ${ }^{-1}\left(\mathrm{~cm}^{2}\right)$, fresh and dry weight $(\mathrm{g})$ of vegetative and root parts as well as root length $(\mathrm{cm})$. and the percentage of survival plants was calculated.

\section{Flowering measurements:}

When the flowers were full open, number of flowers plant ${ }^{-1}$, diameter of flower $(\mathrm{cm})$, fresh and dry weights of flowersplant ${ }^{-1}$ were determined.

\section{Nutritional status:}

Twenty mature leaves plant $^{-1}\left(5^{\text {th }}\right.$ fresh leaf) were sampled in both seasons, washed, dried at $70^{\circ} \mathrm{C}$ to a constant weight, ground and digested for determination leaf minerals (nitrogen, phosphorus, potassium, calcium, sodium, and chloride \%). Total nitrogen was determined by micro-Kjeldahle method as summarized by Chapman and Pratt (1978). Phosphorus was determined using spectrophotometer according to Murphy and Riely (1962). Potassium and sodium were determined by flame photometer as described by Jackson (1967), Brown and Lilleland (1946). Chloride was determined according to Kraemer and Stamm (1924). Calcium was determined as described by Cheng and Bray (1951). Carotene contents in flowers were determined as mentioned by Nagata and Yamashita (1992). Proline was assessed colorimetric ally in fresh samples as illustrated by Bates et al., (1973). Total green color was determined in mature leaves by using MINOLTA CHLOROPHYLL METER SPAD-502 (Minolta camera. Co, Ltd Japan) (Wood et al., 1993).

\section{Statistical Analysis:}

The mean and ANOVA were analyzed using MSTATC computer software program (Bricker, 1991). The obtained data were subjected to analysis of variance according Snedecor and Cochran (1990). Duncan's multiple range test (Duncan, 1955) for significance was determined at $p<0.05$.

\section{RESULTS AND DISCUSSION}

\section{1- Vegetative and root growth: \\ a-Vegetative growth:}

Results presented in Tables (4 and 5) revealed that irrigation by diluted seawater at 10,20 and $30 \%$ seawater significantly decreased all vegetative growth parameters (plant height, number of shoots plant-1, number of leaves/plant, total leaf area plant-1, plant fresh and dry weight) compared with control (tap water) in both seasons. The highest values were obtained by control followed in descending order to 10, 20 and $30 \%$ seawater except for the parameter of dry weight plant-1 in the second season only which gave the highest values by control and diluted seawater at $10 \%$ treatments without significant differences in between. Reducing vegetative growth parameters by high salinity may be associated with the reducing of turgor pressure and the high energy consumed of massive salt secretion and osmoregulation. Moreover, excess salt decreases the leaf water potential, as in water deficit conditions, reduces water and nutrients uptake by plants, and finally leads to decrease the growth (Xiao-Hua et al., 2009). Also, abiotic stresses counting salt-stress induce accumulation of reactive oxygen species (ROS) that are harmful to cells at high concentrations as they cause the oxidative injury to membrane lipids, proteins, and nucleic acids ( Ashraf and Harris, 2004). The obtained results agree with those 
reported by Hashish et al., (2015), Nofal et al., (2015) and Abou El-Ftouh et al., (2018) on Calendula plants. In addition, ElMahrouk et al., (2010) on buttonwood plants, Turhan et al., (2014) on lettuce, Bafeel et al., (2016) on Jojoba plants and
El- Sayed et al., (2017), on Duranta plumieri. They indicated that the plant growth was negatively correlated with increasing saline water levels. The values of growth parameters were reduced with increasing salinity levels.

Table (4): Effect of diluted seawater irrigation, salicylic acid spray levels and their interaction on plant height and No. of shoots and leaves plant ${ }^{-1}$ of Calendula $^{-}$ officinalis L. during 2017/2018 and 2018/2019 seasons

\begin{tabular}{|c|c|c|c|c|c|c|c|}
\hline \multirow{2}{*}{\multicolumn{2}{|c|}{ Treatments }} & \multicolumn{2}{|c|}{ Plant height (cm) } & \multicolumn{2}{|c|}{ No. of shoots plant ${ }^{-1}$} & \multicolumn{2}{|c|}{ No. of leaves plant ${ }^{-1}$} \\
\hline & & $\begin{array}{c}1 \underline{\text { st }} \\
\text { season }\end{array}$ & $2^{\text {nd }}$ season & 1st season & $2^{\text {nd }}$ season & 1st season & $\begin{array}{c}2^{\text {nd }} \\
\text { season }\end{array}$ \\
\hline \multicolumn{8}{|c|}{ Seawater (\%) } \\
\hline \multicolumn{2}{|c|}{ Cont. } & $47.22 \mathrm{a}$ & $46.44 a$ & $10.78 a$ & $10.22 a$ & 279.00a & $275.78 a$ \\
\hline \multicolumn{2}{|c|}{$10 \%$} & $43.56 b$ & 43.33b & $9.22 b$ & $8.78 b$ & 257.44b & 260.78b \\
\hline \multicolumn{2}{|c|}{$20 \%$} & $40.33 c$ & $40.11 c$ & $7.67 \mathrm{c}$ & 7.22c & $169.33 c$ & $172.67 \mathrm{c}$ \\
\hline \multicolumn{2}{|c|}{$30 \%$} & $36.11 d$ & $36.00 \mathrm{~d}$ & $6.44 d$ & $7.00 \mathrm{c}$ & $151.22 d$ & $152.33 d$ \\
\hline \multicolumn{8}{|c|}{ Salicylic acid (ppm) } \\
\hline \multicolumn{2}{|c|}{0} & 38.42c & $37.75 c$ & $7.50 \mathrm{c}$ & $7.25 b$ & 198.67c & 198.67b \\
\hline \multicolumn{2}{|c|}{150} & 41.75b & 41.00b & $8.50 \mathrm{~b}$ & $8.42 a$ & 218.50b & $220.67 a$ \\
\hline \multicolumn{2}{|c|}{300} & $45.25 a$ & $45.67 a$ & $9.58 a$ & $9.25 a$ & $225.58 a$ & $226.83 a$ \\
\hline Seawater & $\begin{array}{c}\text { SA } \\
(p p m)\end{array}$ & \multicolumn{6}{|c|}{ Interaction } \\
\hline \multirow{3}{*}{ Cont. } & 0 & $45.00 \mathrm{bcd}$ & 43.67cd & $9.67 \mathrm{bcd}$ & 9.33abcd & 259.67b & 258.67c \\
\hline & 150 & 46.33bc & $46.00 \mathrm{bc}$ & 10.67b & $10.33 a b$ & $285.00 a$ & 278.33ab \\
\hline & 300 & $50.33 a$ & $49.67 a$ & $12.00 \mathrm{a}$ & $11.00 \mathrm{a}$ & 292.33a & $290.33 a$ \\
\hline \multirow{3}{*}{$10 \%$} & 0 & 39.33fg & $39.67 e$ & 8.00ef & 7.33efg & 240.67c & $242.00 d$ \\
\hline & 150 & $43.33 \mathrm{cde}$ & 42.67d & $9.33 \mathrm{~cd}$ & $9.00 \mathrm{bcde}$ & 262.33b & $270.33 b c$ \\
\hline & 300 & 48.00ab & 47.67ab & $10.33 \mathrm{bc}$ & $10.00 \mathrm{abc}$ & 269.33b & $270.00 \mathrm{bc}$ \\
\hline \multirow{3}{*}{$20 \%$} & 0 & $37.33 \mathrm{~g}$ & 35.33f & 6.67ghi & $6.33 \mathrm{~g}$ & $154.67 \mathrm{e}$ & $158.00 \mathrm{~g}$ \\
\hline & 150 & 40.67ef & $39.67 \mathrm{e}$ & 7.67efg & $7.00 \mathrm{fg}$ & $173.00 d$ & 176.33ef \\
\hline & 300 & 43.00de & 45.33bc & $8.67 \mathrm{de}$ & 8.33cdef & $180.33 d$ & $183.67 e$ \\
\hline \multirow{3}{*}{$30 \%$} & 0 & 32.00h & $32.33 \mathrm{~g}$ & $5.67 i$ & $6.00 \mathrm{~g}$ & $139.67 f$ & 136.00h \\
\hline & 150 & $36.67 \mathrm{~g}$ & $35.67 f$ & 6.33hi & 7.33efg & $153.67 \mathrm{e}$ & $157.67 \mathrm{~g}$ \\
\hline & 300 & $39.67 f g$ & $40.00 \mathrm{e}$ & 7.33fgh & 6.67defg & $160.33 \mathrm{e}$ & $163.33 \mathrm{fg}$ \\
\hline
\end{tabular}

Means within a column having the same letters are not significantly differences according to Duncan's multiple range test (DMRT). 
Table (5): Effect of diluted seawater irrigation, salicylic acid spray levels and their interaction on total leaf area and fresh and dry weight plant ${ }^{-1}$ of Calendula officinalis L. during 2017/2018 and 2018/2019 seasons

\begin{tabular}{|c|c|c|c|c|c|c|c|}
\hline \multirow{2}{*}{\multicolumn{2}{|c|}{ Treatments }} & \multicolumn{2}{|c|}{ Total leaf area $\left(\mathrm{cm}^{2}\right)$} & \multicolumn{2}{|c|}{$\begin{array}{l}\text { Fresh weight plant }{ }^{-1} \\
(\mathrm{gm})\end{array}$} & \multicolumn{2}{|c|}{$\begin{array}{l}\text { Dry weight plant }{ }^{-1} \\
\text { (gm) }\end{array}$} \\
\hline & & 1st season & $2^{\text {nd }}$ season & 1st season & $2^{\text {nd }}$ season & 1st season & $2^{\text {nd }}$ season \\
\hline \multicolumn{8}{|c|}{ Seawater (\%) } \\
\hline \multicolumn{2}{|c|}{ Cont. } & $5252 a$ & $5292.26 a$ & $251.78 a$ & $259.79 a$ & $33.35 a$ & $34.77 a$ \\
\hline \multicolumn{2}{|c|}{$10 \%$} & $4667.67 b$ & $4642.73 b$ & $229.65 b$ & $241.34 b$ & $32.21 b$ & $34.13 a$ \\
\hline \multicolumn{2}{|c|}{$20 \%$} & $2791.83 c$ & $2892.96 c$ & $206.81 c$ & $214.80 c$ & $30.41 \mathrm{c}$ & $31.92 b$ \\
\hline \multicolumn{2}{|c|}{$30 \%$} & $2342.98 d$ & $2337.66 d$ & $176.96 d$ & $184.15 d$ & 27.44d & $28.63 \mathrm{c}$ \\
\hline \multicolumn{8}{|c|}{ Salicylic acid (ppm) } \\
\hline \multicolumn{2}{|c|}{0} & $3265.09 c$ & $3320.15 c$ & $206.66 c$ & 213.81c & $28.44 \mathrm{c}$ & 29.71c \\
\hline \multicolumn{2}{|c|}{150} & $3884.44 b$ & $3868.17 b$ & 213.99b & $221.41 b$ & $30.74 b$ & $31.98 b$ \\
\hline \multicolumn{2}{|c|}{300} & $4141.82 a$ & $4185.88 a$ & $228.26 a$ & $239.83 a$ & $33.39 a$ & $35.39 a$ \\
\hline Seawater & $\begin{array}{c}\text { SA } \\
\text { (ppm) }\end{array}$ & \multicolumn{6}{|c|}{ Interaction } \\
\hline \multirow{3}{*}{ Cont. } & 0 & $4639.72 d$ & $4837.29 c$ & $247.90 b$ & $250.50 b$ & $31.72 \mathrm{~cd}$ & $32.45 d$ \\
\hline & 150 & $5329.28 b$ & $5270.75 b$ & $249.47 b$ & $254.27 b$ & $33.26 \mathrm{~b}$ & $33.86 \mathrm{c}$ \\
\hline & 300 & $5788.92 a$ & $5768.74 a$ & $257.98 a$ & $274.60 a$ & $35.06 a$ & $37.98 \mathrm{a}$ \\
\hline \multirow{3}{*}{$10 \%$} & 0 & $4090.17 e$ & $4108.47 d$ & $220.80 \mathrm{e}$ & $230.80 d$ & $29.92 \mathrm{e}$ & $31.55 d$ \\
\hline & 150 & $4900.00 \mathrm{~cd}$ & $4769.75 c$ & 228.17d & $239.88 c$ & $32.40 \mathrm{bc}$ & $34.18 \mathrm{c}$ \\
\hline & 300 & $5012.84 c$ & $5049.97 b c$ & $240.00 c$ & $253.33 b$ & $34.32 a$ & $36.65 b$ \\
\hline \multirow{3}{*}{$20 \%$} & 0 & 2358.20h & $2488.94 \mathrm{~g}$ & $195.84 \mathrm{~g}$ & $202.48 f$ & $27.67 f$ & $28.89 \mathrm{e}$ \\
\hline & 150 & $2870.34 \mathrm{fg}$ & 2946.70f & $206.77 f$ & $215.67 \mathrm{e}$ & 30.82de & $32.49 d$ \\
\hline & 300 & $3146.95 f$ & $3243.24 \mathrm{e}$ & $217.82 e$ & $226.24 d$ & $32.75 b$ & $34.38 \mathrm{c}$ \\
\hline \multirow{3}{*}{$30 \%$} & 0 & $1972.25 i$ & 1845.90h & $162.11 \mathrm{i}$ & $171.45 \mathrm{~g}$ & $24.43 h$ & $25.95 \mathrm{~g}$ \\
\hline & 150 & $2438.12 h$ & $2485.49 \mathrm{~g}$ & $171.53 \mathrm{~h}$ & $175.84 \mathrm{~g}$ & $26.46 \mathrm{~g}$ & $27.39 f$ \\
\hline & 300 & 2618.59gh & 2681.58fg & $197.25 \mathrm{~g}$ & $205.15 f$ & $31.43 \mathrm{~cd}$ & $32.56 \mathrm{~d}$ \\
\hline
\end{tabular}

Means within a column having the same letters are not significantly differences according to Duncan's multiple range test (DMRT).

Regarding salicylic acid (SA) treatments, all tested foliar application significantly increased all vegetative growth parameters (plant height, number of shoots plant-1, number of leaves plant1 , total leaf area plant-1, plant fresh and dry weight) compared with the control treatment in both seasons. Salicylic acid at $300 \mathrm{ppm}$ gave the highest values followed by salicylic at 150ppm. In general, under the same saline water irrigation concentration, spraying salicylic acid alleviated the harmful effects of saline water irrigation on the plants and enhanced the plant growth especially at $300 \mathrm{ppm}$ as compared with 
control treatment in both seasons. The enhancement in growth parameters by applying SA may be due to enhancing ions absorption and minerals by plant. Moreover, improves plant performance by formation of certain enzymes in plant, hence stimulating chlorophyll synthesis and photosynthetic activities, which improve plant growth (Hayat et al., 2007). In addition, SA plays a main role in plant growth regulation and development is a hormone-like substance (Raskin, 1992) which have defensive effects in contrast to abiotic stress factors such as salinity and heat (Strobel and Kuc, 1995). These results are similar with those of Hashish et al., (2015) on Calendula officinalis and Basit et al., (2018) on Tagetes sp. L.

The interaction of salinity (irrigation by diluted seawater) and salicylic acid on vegetative growth parameters of calendula plants are shown in Tables (4 and 5). All growth parameters were affected by the interaction compared with control plants. Plants watered with tap water (0\% seawater) and spraying by $\mathbf{3 0 0}$ ppm SA followed by plants irrigated with diluted seawater at $10 \%$ and were sprayed with SA at $300 \mathrm{ppm}$ showed increased all growth parameters compared with the other treatments. In this respect, it can be assume that the depressing effects of salinity on plant growth and other relevant physiological activities can be alleviated by spraying plants by the appropriate concentrations of salicylic acid (Afzal et al., 2006). The ameliorative effects of SA have been well documented in inducing salt tolerance in many plants. Moreover, salicylic acid is an endogenous growth regulator of phenol nature, which participates in the regulation of physiological processes in plants. It plays an important role in the plant response environmental conditions such as salinity (Baby et al., 2010). The inhibition effects of salinity on growth parameters might be due to salinity which inhibits of growth through reduced water absorption, reduced metabolic activities due to $\mathrm{Na}+$ and $\mathrm{Cl}$ toxicity and nutrient deficiency caused by ionic interference (Delacerda et al.,2003). The obtained results show similarity to findings by Hashish et al., (2015), Nofal et al., (2015) and Abou ElFtouh et al., (2018) on Calendula plants.

\section{b-Root growth:}

Data registered in Table (6) indicated that root growth parameters (root length and fresh and dry weights) were significantly gradually decreased by increasing diluted seawater irrigation levels from 10 to $30 \%$ comparing with the tap water ( $0 \%$ seawater) in both seasons. The decreasing in growth parameters of roots attributable to salinity might be due to the decline in water and minerals absorption or the reduction in upper ground plant parts (Mazhar et al., 2006). These results are in harmony with those of Nofal et al., (2015) and Abou El-Ftouh et al., (2018) on Calendula plants and ElMahrouk et al., (2010) on buttonwood plants. With regard the effect of salicylic acid (SA) treatments on Calendula plants, data presented in Table (6) emphasized that foliar application of SA significantly promoted all root growth parameters compared with control. The highest values of root length, fresh and dry weight of roots were recorded by SA treatment at $300 \mathrm{ppm}$ in the first season and SA at 150 and $300 \mathrm{ppm}$ without significant differences in between. As for the interaction between both factors (diluted seawater irrigation $x S A$ ) the highest values of root parameters were obtained by the combination treatments of tap water ( $0 \%$ seawater) $\times \mathrm{SA}$ at $300 \mathrm{ppm}$ and diluted seawater at $10 \% \times$ SA at $\mathbf{3 0 0}$ ppm compared with the other treatments. These results are in harmony with those of Pacheco et al., (2013), Hashish et al., (2015) and Abou El-Ftouh et al., (2018) on Calendula officinalis. They revealed that foliar applications of SA increased root growth characteristics (fresh and dry weights of roots and roots volume). 
Table (6): Effect of diluted seawater irrigation, salicylic acid spray levels and their interaction on root length, root fresh and dry weight of Calendula officinalis $\mathrm{L}$. during 2017/2018 and 2018/2019 seasons

\begin{tabular}{|c|c|c|c|c|c|c|c|}
\hline \multirow{2}{*}{\multicolumn{2}{|c|}{ Treatments }} & \multicolumn{2}{|c|}{ Root length (cm) } & \multicolumn{2}{|c|}{$\begin{array}{l}\text { Root fresh weight } \\
\text { (gm) }\end{array}$} & \multicolumn{2}{|c|}{ Root dry weight (gm) } \\
\hline & & $\begin{array}{l}1 \underline{\text { st }} \\
\text { season }\end{array}$ & $\begin{array}{l}2^{\text {nd }} \\
\text { season }\end{array}$ & $\begin{array}{l}1{ }^{\text {st }} \\
\text { season }\end{array}$ & $\begin{array}{l}2 \stackrel{\text { nd }}{\text { season }} \\
\text { seas }\end{array}$ & $\begin{array}{c}1 \underline{\text { st }} \\
\text { season }\end{array}$ & $\begin{array}{c}2^{\text {nd }} \\
\text { season }\end{array}$ \\
\hline \multicolumn{8}{|c|}{ Seawater (\%) } \\
\hline \multicolumn{2}{|c|}{ Cont. } & $26.00 a$ & 27.11a & $22.72 a$ & $25.21 a$ & $4.59 a$ & $5.07 a$ \\
\hline \multicolumn{2}{|c|}{$10 \%$} & $21.33 b$ & $22.44 b$ & $20.02 b$ & $21.64 b$ & $4.15 b$ & $4.48 b$ \\
\hline \multicolumn{2}{|c|}{$20 \%$} & $17.11 \mathrm{c}$ & $18.11 \mathrm{c}$ & $15.95 \mathrm{c}$ & $16.70 \mathrm{c}$ & $3.40 \mathrm{c}$ & $3.61 \mathrm{c}$ \\
\hline \multicolumn{2}{|c|}{$30 \%$} & $13.33 d$ & $14.00 \mathrm{~d}$ & $12.81 d$ & $14.06 \mathrm{~d}$ & 2.84d & $3.13 d$ \\
\hline \multicolumn{8}{|c|}{ Salicylic acid (ppm) } \\
\hline \multicolumn{2}{|c|}{0} & $18.33 b$ & $19.25 b$ & $16.29 \mathrm{c}$ & $17.73 \mathrm{c}$ & $3.40 \mathrm{c}$ & $3.69 \mathrm{c}$ \\
\hline \multicolumn{2}{|c|}{150} & $19.33 b$ & 20.42ab & $17.80 \mathrm{~b}$ & $19.19 b$ & $3.70 a$ & $4.00 \mathrm{~b}$ \\
\hline \multicolumn{2}{|c|}{300} & $20.67 a$ & $21.58 a$ & $19.53 a$ & $21.28 a$ & $4.14 a$ & $4.53 a$ \\
\hline Seawater & $\begin{array}{c}\mathrm{SA} \\
(\mathrm{ppm})\end{array}$ & \multicolumn{6}{|c|}{ Interaction } \\
\hline \multirow{3}{*}{ Cont. } & 0 & $24.67 b$ & $25.67 b$ & $21.21 \mathrm{c}$ & $23.49 \mathrm{c}$ & $4.32 \mathrm{c}$ & $4.73 c$ \\
\hline & 150 & 26.00ab & $27.33 a$ & $22.78 b$ & $25.18 b$ & $4.59 b$ & 5.07b \\
\hline & 300 & $27.33 a$ & $28.33 a$ & $24.15 a$ & $26.95 a$ & $4.87 a$ & $5.43 a$ \\
\hline \multirow{3}{*}{$10 \%$} & 0 & 20.33d & 21.33d & $19.22 \mathrm{~d}$ & 20.91d & $3.96 \mathrm{~d}$ & $4.29 d$ \\
\hline & 150 & $21.00 \mathrm{~d}$ & 22.33d & $19.44 d$ & 21.23d & 3.99d & $4.36 d$ \\
\hline & 300 & $22.67 \mathrm{c}$ & $23.67 \mathrm{c}$ & $21.41 \mathrm{c}$ & $22.77 \mathrm{c}$ & $4.50 \mathrm{bc}$ & $4.79 c$ \\
\hline \multirow{3}{*}{$20 \%$} & 0 & $16.00 \mathrm{fg}$ & $17.00 \mathrm{f}$ & $14.22 \mathrm{~g}$ & $14.53 \mathrm{~g}$ & $2.99 f$ & $3.09 f$ \\
\hline & 150 & $17.33 \mathrm{ef}$ & $18.33 \mathrm{e}$ & $16.00 \mathrm{f}$ & $16.32 \mathrm{f}$ & $3.37 \mathrm{e}$ & $3.48 \mathrm{e}$ \\
\hline & 300 & $18.00 \mathrm{e}$ & $19.00 \mathrm{e}$ & $17.92 \mathrm{e}$ & $19.24 \mathrm{e}$ & $3.85 d$ & $4.25 d$ \\
\hline \multirow{3}{*}{$30 \%$} & 0 & $12.33 \mathrm{~h}$ & $13.00 \mathrm{~h}$ & $10.51 \mathrm{i}$ & $11.99 \mathrm{~h}$ & $2.31 \mathrm{~g}$ & $2.67 \mathrm{~g}$ \\
\hline & 150 & $13.00 \mathrm{~h}$ & $13.67 \mathrm{~h}$ & $12.98 \mathrm{~h}$ & $14.03 \mathrm{~g}$ & $2.85 f$ & $3.07 f$ \\
\hline & 300 & $14.67 \mathrm{~g}$ & $15.33 \mathrm{~g}$ & $14.93 \mathrm{fg}$ & $16.17 f$ & $3.36 \mathrm{e}$ & $3.64 \mathrm{e}$ \\
\hline
\end{tabular}

Means within a column having the same letters are not significantly differences according to Duncan's multiple range test (DMRT).

\section{2- Flowering measurements:}

Data presented in Table (7) showed that diluted seawater irrigation treatments significantly decreased all flowering parameters (number of flowers, diameter, flower fresh and dry weight) compared with the irrigation by tap water treatment (control). The highest values in these parameters number of flowers (21.5 and
20.44), flower diameter ( 4.50 and $4.61 \mathrm{~cm})$, flower fresh weight (28.87 and 28.99) and dry weight (3.74 and $3.95 \mathrm{~g})$ were recorded by the control followed by the treatment of diluted seawater irrigation at $10 \%$ seawater which recorded the second rank in number of flowers (16.22 and 15.44), diameter (4.17 and $4.06 \mathrm{~cm})$, flower fresh weight (16.48 and $16.63 \mathrm{~g}$ ) and dry weight (2.33 and $2.41 \mathrm{~g})$, while the lowest 
Influence of foliar application of salicylic acid on growth and flowering .........

values of number of flowers $(7.00$ and 6.22), flower diameter ( 3.44 and $3.39 \mathrm{~cm})$, flower fresh weight $(5.92$ and $5.44 \mathrm{~g})$ and dry weight $(0.81$ and $0.82 \mathrm{~g})$ were obtained by the treatment of seawater irrigation at $30 \%$ seawater in both seasons, respectively. The reduction in flowering parameters may ensue from the plants inability to adjust somatically, counteraction toxicities or related disruptive phenomena or from the excessive energy demand placed upon the metabolic machinery required by such homeostatic systems (Greenway and Munns, 1980). These results are in harmony with those of Hashish et al., (2015) and Abou El-Ftouh et al., (2018) on Calendula officinalis.

Table (7): Effect of diluted seawater irrigation, salicylic acid spray levels and their interaction on No. of flowers plant-1, flower diameter and flower fresh and dry weight of Calendula officinalis L. during 2017/2018 and 2018/2019 seasons.

\begin{tabular}{|c|c|c|c|c|c|c|c|c|c|}
\hline \multirow{2}{*}{\multicolumn{2}{|c|}{ Treatments }} & \multicolumn{2}{|c|}{$\begin{array}{l}\text { No. of flowers } \\
\text { plant }^{-1}\end{array}$} & \multicolumn{2}{|c|}{$\begin{array}{l}\text { Flower diameter } \\
\text { (cm) }\end{array}$} & \multicolumn{2}{|c|}{$\begin{array}{c}\text { Flower fresh } \\
\text { weight (g) }\end{array}$} & \multicolumn{2}{|c|}{$\begin{array}{l}\text { Flower dry } \\
\text { weight (g) }\end{array}$} \\
\hline & & $\begin{array}{c}1{ }^{\text {st }} \\
\text { season }\end{array}$ & $\begin{array}{c}2^{\text {nd }} \\
\text { season }\end{array}$ & $\begin{array}{c}1 \underline{\text { st }} \\
\text { season }\end{array}$ & $\begin{array}{c}2^{\text {nd }} \\
\text { season }\end{array}$ & $\begin{array}{c}1^{\text {st }} \\
\text { season }\end{array}$ & $\begin{array}{c}2^{\text {nd }} \\
\text { season }\end{array}$ & $\begin{array}{c}1 \underline{\text { st }} \\
\text { season }\end{array}$ & $\begin{array}{c}2^{\text {nd }} \\
\text { season }\end{array}$ \\
\hline \multicolumn{10}{|c|}{ Seawater (\%) } \\
\hline \multicolumn{2}{|c|}{ Cont. } & $21.56 a$ & $20.44 a$ & $4.50 a$ & $4.61 \mathrm{a}$ & $28.87 a$ & $28.99 a$ & $3.74 a$ & $3.95 a$ \\
\hline \multicolumn{2}{|c|}{$10 \%$} & $16.22 \mathrm{~b}$ & $15.44 b$ & 4.17b & 4.06b & $16.48 b$ & 16.63b & $2.33 b$ & $2.41 b$ \\
\hline \multicolumn{2}{|c|}{$20 \%$} & $10.33 c$ & $9.89 \mathrm{c}$ & $3.78 \mathrm{c}$ & $3.78 b$ & $9.06 \mathrm{c}$ & $9.15 \mathrm{c}$ & $1.38 \mathrm{c}$ & $1.42 \mathrm{c}$ \\
\hline \multicolumn{2}{|c|}{$30 \%$} & $7.00 \mathrm{~d}$ & $6.22 d$ & $3.44 d$ & $3.39 \mathrm{c}$ & $5.92 d$ & $5.44 d$ & $0.81 d$ & $0.82 d$ \\
\hline \multicolumn{10}{|c|}{ Salicylic acid (ppm) } \\
\hline \multicolumn{2}{|c|}{0} & $10.92 \mathrm{c}$ & $10.00 \mathrm{c}$ & $3.79 a$ & $3.79 \mathrm{~b}$ & $11.51 \mathrm{c}$ & $10.91 \mathrm{c}$ & $1.49 \mathrm{c}$ & 1.51c \\
\hline \multicolumn{2}{|c|}{150} & $13.58 \mathrm{~b}$ & $12.75 b$ & $3.92 a$ & $3.88 \mathrm{~b}$ & $14.64 \mathrm{~b}$ & $14.03 b$ & $1.96 \mathrm{~b}$ & $2.07 \mathrm{~b}$ \\
\hline \multicolumn{2}{|c|}{300} & $16.83 a$ & $16.25 a$ & $4.21 a$ & $4.21 a$ & $19.10 \mathrm{a}$ & $20.23 a$ & $2.74 a$ & $2.86 a$ \\
\hline Seawater & $\begin{array}{c}\text { SA } \\
\text { (ppm) }\end{array}$ & \multicolumn{8}{|c|}{ Interaction } \\
\hline \multirow{3}{*}{ Cont. } & 0 & $19.00 \mathrm{c}$ & $17.33 \mathrm{c}$ & $4.17 \mathrm{bc}$ & $4.33 \mathrm{bc}$ & $23.70 \mathrm{c}$ & $22.72 \mathrm{c}$ & $2.86 \mathrm{c}$ & $2.99 \mathrm{c}$ \\
\hline & 150 & $21.67 \mathrm{~b}$ & $20.33 b$ & $4.50 \mathrm{ab}$ & $4.50 \mathrm{ab}$ & $29.02 b$ & $27.74 b$ & $3.64 b$ & 3.84b \\
\hline & 300 & $24.00 \mathrm{a}$ & $23.67 a$ & $4.83 a$ & $5.00 a$ & $33.89 a$ & $36.52 a$ & $4.72 a$ & $5.03 a$ \\
\hline \multirow{3}{*}{$10 \%$} & 0 & $13.67 \mathrm{e}$ & $13.33 d$ & $4.00 \mathrm{bc}$ & $4.00 \mathrm{bcd}$ & $13.48 \mathrm{e}$ & $13.05 d$ & $1.79 \mathrm{e}$ & $1.89 \mathrm{e}$ \\
\hline & 150 & $16.00 \mathrm{~d}$ & $14.67 d$ & $4.17 \mathrm{bc}$ & $4.00 \mathrm{bcd}$ & $15.40 \mathrm{e}$ & $14.20 \mathrm{~d}$ & $2.19 d$ & $2.30 \mathrm{~d}$ \\
\hline & 300 & $19.00 \mathrm{c}$ & $18.33 \mathrm{c}$ & $4.33 b$ & $4.17 \mathrm{bcd}$ & 20.57d & $22.64 c$ & $3.00 \mathrm{c}$ & $3.05 \mathrm{c}$ \\
\hline \multirow{3}{*}{$20 \%$} & 0 & $6.67 \mathrm{~g}$ & $5.33 \mathrm{fg}$ & $3.67 \mathrm{~cd}$ & $3.67 \mathrm{de}$ & $5.49 \mathrm{~g}$ & $4.60 f$ & $0.84 \mathrm{~g}$ & $0.73 \mathrm{~g}$ \\
\hline & 150 & $10.00 f$ & $10.00 \mathrm{e}$ & $3.67 \mathrm{~cd}$ & $3.83 \mathrm{~cd}$ & $8.68 f$ & $8.98 \mathrm{e}$ & $1.30 \mathrm{f}$ & $1.42 f$ \\
\hline & 300 & 14.33de & 14.33d & $4.00 \mathrm{bc}$ & $3.83 \mathrm{~cd}$ & $13.01 \mathrm{e}$ & $13.87 d$ & $2.00 \mathrm{de}$ & $1.42 \mathrm{f}$ \\
\hline \multirow{3}{*}{$30 \%$} & 0 & $4.33 \mathrm{~h}$ & $4.00 \mathrm{~g}$ & $3.33 d$ & $3.17 \mathrm{e}$ & $3.38 \mathrm{~g}$ & $3.25 f$ & $0.48 \mathrm{~h}$ & $0.44 \mathrm{~g}$ \\
\hline & 150 & $6.67 \mathrm{~g}$ & $6.00 f$ & 3.33d & $3.17 e$ & $5.45 \mathrm{~g}$ & $5.18 \mathrm{f}$ & $0.71 \mathrm{gh}$ & $0.74 \mathrm{~g}$ \\
\hline & 300 & $10.00 f$ & 8.67e & $3.67 \mathrm{~cd}$ & $3.83 \mathrm{~cd}$ & $8.93 f$ & $7.90 \mathrm{e}$ & $1.25 f$ & $1.28 f$ \\
\hline
\end{tabular}

Means within a column having the same letters are not significantly differences according to Duncan's multiple range test (DMRT). 
Foliar application of salicylic acid treatments on Calendula officinalis plants significantly increased number of flowers plant-1, flower diameter, flower fresh and dry weight compared with the control. SA at $300 \mathrm{ppm}$ gave the highest significant increases in number of flowers per plant (16.83 and 16.25) in both seasons respectively, meanwhile, there were nonsignificant differences among SA treatments for flower diameter in the first season but in the second one the differences were significant, the highest value $(4.21 \mathrm{~cm})$ was recorded with $S A$ at $300 \mathrm{ppm}$ followed by SA at 150 and $0 \mathrm{ppm}$ without significant differences between themselves. The highest values of flower fresh weight (19.10 and $20.23 \mathrm{~g}$ ) and dry weight (2.74 and $2.86 \mathrm{~g})$ were obtained by $\mathrm{SA}$ at $\mathbf{3 0 0} \mathrm{ppm}$ followed in a descending order by SA at 150 and $0 \mathrm{ppm}$. The highest values of flowering parameters were obtained by the combination of control (tap water) +300 ppm SA. Also $10 \%$ seawater $+300 \mathrm{ppm}$ SA. The positive effect of SA was attributed to enhancing CO2 assimilation, chlorophyll concentration, photosynthetic rate and improved mineral uptake by stressed plants which were treated with SA (Karlidage et al., 2009). In the same line, Pacheco et al., (2013), Hashish et al., (2015) and Basit et al., (2018) on Calendula plant, showed that, foliar application of salicylic acid treatments on marigold plants significantly enhanced the flowering compared with untreated plants.

\section{3- Chemical composition:}

a-Leaf total chlorophyll, carotene contents in flowers and proline content

Data presented in Table (8) illustrated that all tested diluted seawater treatments significantly decreased leaf total chlorophyll (total green color, SPAD) and carotene contents in flowers, while proline content was increased compared with the tap water treatment (control) in both seasons.

The highest values of total green color and carotene were obtained with control treatment (tap water) followed by diluted seawater irrigation treatment at $10 \%$ compared with the lowest values recorded by diluted seawater treatment at $30 \%$. The lowest photosynthetic ability under salt stress condition was due to stomata closure, inhibition of chlorophyll synthesis, a decrease of carboxylase and due to high chlorophyllase activity (Batanouny et al., 1988). Similar response was previously observed in other plant as salinity caused a decrease in pigment content of calendula plants (Hashish et al., 2015 and Abou El-Ftouh et al., 2018).

All tested foliar spraying salicylic acid treatments significantly increased total green color, carotene contents in flowers, proline content compared with the control in both seasons. spraying of SA at 150 and $300 \mathrm{ppm}$ gave the highest values of flower carotenes and proline contents. Such findings showed a similar trend, changes in photosynthetic content due to stomatal inhabitation associated with metabolic factors other than photosynthetic pigments (Arfan et al., 2007). The application of SA resulted in alleviating the effects of salt stress, because, SA application reduced stomata conductance, intercellular $\mathrm{CO}_{2}$ concentration and transpiration. These results coincide with those reported by Hashish et al., (2015) and Basit et al., (2018) on Calendula plant. The interaction of diluted seawater treatment and salicylic acid on total green color, carotene contents in flowers, proline content of Calendula officinalis plants were demonstrated in Table (8). All parameters were affected by the interaction compared with control. The highest significant 
Influence of foliar application of salicylic acid on growth and flowering

values of carotene contents in flowers and total green color (SPAD) were obtained by plants irrigated with tap water and sprayed with SA at 150, $300 \mathrm{ppm}$ with in both seasons. However, the highest significant values of proline content in both seasons were obtained by the combination treatment of diluted seawater irrigation treatment at 20 and $30 \%$ seawater $\times$ SA at $300 \mathrm{ppm}$.

Table (8): Effect of diluted seawater irrigation, salicylic acid spray levels and their interaction on leaf total green color, carotene contents in flowers and proline content of Calendula officinalis L. during 2017/2018 and 2018/2019 seasons

\begin{tabular}{|c|c|c|c|c|c|c|c|}
\hline \multirow{2}{*}{\multicolumn{2}{|c|}{ Treatments }} & \multicolumn{2}{|c|}{$\begin{array}{l}\text { Total green colour } \\
\text { (SPAD) }\end{array}$} & \multicolumn{2}{|c|}{$\begin{array}{l}\text { Carotene contents in } \\
\text { flowers } \\
(\mathrm{mg} / 100 \mathrm{gm})\end{array}$} & \multicolumn{2}{|c|}{ Proline ( $\mu \mathrm{mole} / \mathrm{gFW}$ ) } \\
\hline & & 1st season & 2nd season & 1.t season & $2 \frac{\text { nd }}{}$ season & 1st season & 2으 season \\
\hline \multicolumn{8}{|c|}{ Seawater (\%) } \\
\hline \multicolumn{2}{|c|}{ Cont. } & $27.76 a$ & $29.92 a$ & $1.247 \mathrm{a}$ & $1.252 a$ & $607.81 a$ & $160.09 d$ \\
\hline \multicolumn{2}{|c|}{$10 \%$} & $22.24 b$ & $23.72 b$ & $0.977 b$ & $0.978 b$ & $484.06 b$ & $254.23 c$ \\
\hline \multicolumn{2}{|c|}{$20 \%$} & $20.81 c$ & $22.00 \mathrm{c}$ & $0.892 c$ & $0.896 c$ & $254.06 c$ & $481.50 b$ \\
\hline \multicolumn{2}{|c|}{$30 \%$} & $17.54 d$ & $18.36 \mathrm{~d}$ & $0.792 d$ & $0.794 d$ & $159.71 d$ & $608.22 a$ \\
\hline \multicolumn{8}{|c|}{ Salicylic acid (ppm) } \\
\hline \multicolumn{2}{|l|}{0} & 21.11c & $22.34 \mathrm{c}$ & $0.915 c$ & $0.919 c$ & $380.55 a$ & $381.02 a$ \\
\hline \multicolumn{2}{|c|}{150} & $22.22 b$ & $23.46 b$ & $0.988 b$ & $0.990 \mathrm{~b}$ & $389.04 a$ & $389.25 a$ \\
\hline \multicolumn{2}{|c|}{300} & $22.94 a$ & $24.70 a$ & $1.028 a$ & $1.031 \mathrm{a}$ & $357.38 a$ & $357.77 a$ \\
\hline Seawater & $\begin{array}{c}\text { SA } \\
(\mathrm{ppm})\end{array}$ & \multicolumn{6}{|c|}{ Interaction } \\
\hline \multirow{3}{*}{ Cont. } & 0 & $26.77 b$ & $28.53 b$ & $1.130 \mathrm{c}$ & $1.131 \mathrm{c}$ & $153.20 \mathrm{~g}$ & $153.37 \mathrm{~g}$ \\
\hline & 150 & $27.27 b$ & $29.60 b$ & $1.250 \mathrm{~b}$ & $1.253 b$ & $160.67 \mathrm{fg}$ & $161.00 \mathrm{fg}$ \\
\hline & 300 & $29.33 a$ & $31.63 a$ & $1.360 \mathrm{a}$ & $1.370 a$ & 165.27efg & $165.90 \mathrm{efg}$ \\
\hline \multirow{3}{*}{$10 \%$} & 0 & $21.40 \mathrm{~d}$ & 22.43def & $0.954 f$ & $0.955 \mathrm{e}$ & $247.00 \mathrm{def}$ & 247.37def \\
\hline & 150 & $22.47 c$ & $23.87 \mathrm{~cd}$ & $0.988 \mathrm{e}$ & $0.986 d$ & $255.00 \mathrm{de}$ & 255.33de \\
\hline & 300 & $22.87 \mathrm{c}$ & $24.87 \mathrm{c}$ & $0.990 \mathrm{~d}$ & $0.992 d$ & $260.17 d$ & $260.00 d$ \\
\hline \multirow{3}{*}{$20 \%$} & 0 & $19.80 \mathrm{e}$ & $21.17 f$ & $0.866 i$ & $0.875 \mathrm{~g}$ & $538.00 \mathrm{~b}$ & $538.67 b$ \\
\hline & 150 & $21.27 d$ & 22.10ef & $0.900 \mathrm{~h}$ & $0.904 f$ & $540.17 b$ & $540.33 b$ \\
\hline & 300 & $21.37 d$ & 22.73de & $0.910 \mathrm{~g}$ & $0.910 f$ & $365.00 \mathrm{c}$ & $365.50 \mathrm{c}$ \\
\hline \multirow{3}{*}{$30 \%$} & 0 & $16.47 \mathrm{~g}$ & $17.23 \mathrm{~h}$ & $0.710 \mathrm{I}$ & $0.714 j$ & $584.00 \mathrm{ab}$ & $584.67 \mathrm{ab}$ \\
\hline & 150 & $17.87 f$ & $18.27 \mathrm{gh}$ & $0.815 k$ & $0.816 i$ & $600.33 a b$ & $600.33 a b$ \\
\hline & 300 & $18.30 f$ & $19.57 \mathrm{~g}$ & $0.851 \mathrm{j}$ & $0.851 \mathrm{~h}$ & $639.11 a$ & $639.67 a$ \\
\hline
\end{tabular}

Means within a column having the same letters are not significantly differences according to Duncan's multiple range test (DMRT). 
b- Leaf mineral ( $\mathrm{N}, \mathrm{P}, \mathrm{K}, \mathrm{Ca}, \mathrm{Na}$ and $\mathrm{Cl})$ and survival \%:

Data presented in Tables (9 and 10) displayed that all tested diluted seawater irrigation treatments significantly decreased nitrogen, phosphorus, potassium and survival $\%$ whereas, sodium and chloride were significantly increased in both seasons. Also, the ratio of $\mathrm{Na} / \mathrm{K}$ was increased with increasing the percentage of diluted seawater from 0 to
$30 \%$ seawater. Salt stress of seawater irrigation led to direct increase in sodium content of aerial parts and the increase in sodium content resulted an increase in $\mathrm{Na} / \mathrm{K}$ ratio in the aerial parts of buttonwood plants (El-Mahrouk et al., 2010). In the same line were the findings of Hussein et al., (2010) on millet and Turhan et al., (2014) on lettuce plants and Abou El-Ftouh et al., (2018) on calendula plant.

Table (9): Effect of diluted seawater irrigation, salicylic acid spray and their interaction on leaf N, P, K and Ca content of Calendula officinalis L. during 2017/2018 and 2018/2019 seasons

\begin{tabular}{|c|c|c|c|c|c|c|c|c|c|}
\hline \multirow{2}{*}{\multicolumn{2}{|c|}{ Treatments }} & \multicolumn{2}{|c|}{$\mathrm{N} \%$} & \multicolumn{2}{|r|}{$\mathbf{P} \%$} & \multicolumn{2}{|c|}{ K \% } & \multicolumn{2}{|c|}{ Ca \% } \\
\hline & & $\begin{array}{c}1 \text { st } \\
\text { season }\end{array}$ & $\begin{array}{c}\text { 2吕 } \\
\text { season }\end{array}$ & $\begin{array}{c}1 \text { st } \\
\text { season }\end{array}$ & $\begin{array}{c}2^{\text {nd }} \\
\text { season }\end{array}$ & $\begin{array}{c}1 \underline{\text { st }} \\
\text { season }\end{array}$ & $\begin{array}{c}2 \frac{\text { nd }}{} \\
\text { season }\end{array}$ & $\begin{array}{c}1 \underline{\text { st }} \\
\text { season }\end{array}$ & $\begin{array}{c}2 \frac{\text { nd }}{} \\
\text { season }\end{array}$ \\
\hline \multicolumn{10}{|c|}{ Seawater (\%) } \\
\hline \multicolumn{2}{|c|}{ Cont. } & $1.49 a$ & $1.50 \mathrm{a}$ & $0.181 a$ & $0.181 \mathrm{a}$ & $1.59 a$ & $1.60 \mathrm{a}$ & $2.29 a$ & $2.31 \mathrm{a}$ \\
\hline \multicolumn{2}{|c|}{$10 \%$} & $1.43 b$ & $1.46 \mathrm{~b}$ & $0.177 b$ & $0.178 b$ & $1.56 \mathrm{~b}$ & $1.57 b$ & $2.20 \mathrm{~b}$ & $2.45 b$ \\
\hline \multicolumn{2}{|c|}{$20 \%$} & $1.33 \mathrm{c}$ & $1.34 \mathrm{c}$ & $0.172 b$ & $0.173 c$ & $1.49 \mathrm{c}$ & $1.50 \mathrm{c}$ & $2.15 \mathrm{c}$ & $2.20 \mathrm{c}$ \\
\hline \multicolumn{2}{|c|}{$30 \%$} & $1.25 d$ & $1.26 \mathrm{~d}$ & $0.164 b$ & $0.164 d$ & $1.45 d$ & $1.46 \mathrm{~d}$ & 2.11d & $2.16 \mathrm{~d}$ \\
\hline \multicolumn{10}{|c|}{ Salicylic acid (ppm) } \\
\hline \multicolumn{2}{|l|}{0} & $1.31 \mathrm{c}$ & $1.32 \mathrm{c}$ & $0.158 \mathrm{c}$ & $0.158 \mathrm{c}$ & $1.47 \mathrm{c}$ & $1.48 \mathrm{c}$ & $2.05 b$ & $2.15 \mathrm{c}$ \\
\hline \multicolumn{2}{|c|}{150} & $1.36 \mathrm{~b}$ & $1.37 b$ & $0.171 b$ & $0.171 b$ & $1.50 \mathrm{~b}$ & $1.51 b$ & $2.26 a$ & $2.25 b$ \\
\hline \multicolumn{2}{|c|}{300} & $1.47 a$ & $1.48 a$ & $0.193 a$ & $0.194 a$ & $1.60 a$ & $1.62 a$ & $2.25 a$ & $2.28 a$ \\
\hline Seawater & $\begin{array}{c}\text { SA } \\
\text { (ppm) }\end{array}$ & \multicolumn{8}{|c|}{ Interaction } \\
\hline \multirow{3}{*}{ Cont. } & 0 & $1.45 \mathrm{c}$ & $1.46 \mathrm{c}$ & $0.166 \mathrm{~h}$ & $0.167 \mathrm{~h}$ & $1.52 \mathrm{e}$ & $1.53 d$ & $2.23 \mathrm{bc}$ & $2.24 \mathrm{c}$ \\
\hline & 150 & $1.46 \mathrm{c}$ & $1.46 \mathrm{c}$ & $0.179 \mathrm{e}$ & $0.179 \mathrm{e}$ & $1.55 \mathrm{c}$ & $1.56 \mathrm{c}$ & $2.33 a$ & $2.37 a$ \\
\hline & 300 & $1.56 \mathrm{a}$ & $1.56 a$ & $0.198 a$ & $0.199 a$ & $1.70 a$ & $1.71 \mathrm{a}$ & $2.31 a$ & $2.31 b$ \\
\hline \multirow{3}{*}{$10 \%$} & 0 & $1.41 \mathrm{e}$ & $1.45 \mathrm{~cd}$ & $0.163 i$ & $0.164 i$ & $1.50 \mathrm{f}$ & $1.51 \mathrm{e}$ & $2.10 \mathrm{~d}$ & $2.23 c$ \\
\hline & 150 & $1.40 \mathrm{~d}$ & $1.42 \mathrm{de}$ & $0.172 f$ & $0.173 f$ & $1.50 \mathrm{f}$ & $1.51 \mathrm{e}$ & $2.25 b$ & $2.26 \mathrm{bc}$ \\
\hline & 300 & $1.49 \mathrm{~b}$ & $1.51 \mathrm{~b}$ & $0.196 b$ & $0.197 b$ & $1.69 b$ & $1.70 \mathrm{~b}$ & $2.23 b c$ & $2.24 \mathrm{c}$ \\
\hline \multirow{3}{*}{$20 \%$} & 0 & $1.25 \mathrm{~h}$ & $1.26 \mathrm{~g}$ & $0.155 k$ & $0.155 k$ & $1.45 i$ & $1.46 \mathrm{~g}$ & $2.00 \mathrm{e}$ & $2.13 d$ \\
\hline & 150 & $1.31 \mathrm{~g}$ & $1.32 \mathrm{f}$ & $0.170 \mathrm{~g}$ & $0.172 \mathrm{~g}$ & $1.48 \mathrm{~h}$ & $1.49 \mathrm{f}$ & $2.23 \mathrm{bc}$ & $2.24 \mathrm{c}$ \\
\hline & 300 & $1.42 d$ & $1.44 \mathrm{~cd}$ & $0.190 c$ & $0.192 c$ & $1.54 d$ & $1.55 \mathrm{c}$ & $2.22 \mathrm{bc}$ & $2.23 c$ \\
\hline \multirow{3}{*}{$30 \%$} & 0 & $1.12 \mathrm{i}$ & $1.13 \mathrm{~h}$ & $0.145 I$ & $0.146 \mathrm{I}$ & $1.40 \mathrm{j}$ & $1.41 \mathrm{~h}$ & $1.90 \mathrm{f}$ & $2.00 \mathrm{e}$ \\
\hline & 150 & $1.25 \mathrm{~h}$ & $1.26 \mathrm{~g}$ & $0.160 \mathrm{j}$ & $0.161 \mathrm{j}$ & $1.47 \mathrm{~g}$ & $1.47 \mathrm{~g}$ & $2.22 \mathrm{bc}$ & $2.26 \mathrm{bc}$ \\
\hline & 300 & $1.39 f$ & $1.40 \mathrm{e}$ & $0.185 d$ & $0.186 d$ & $1.48 \mathrm{~g}$ & $1.50 f$ & $2.21 \mathrm{c}$ & $2.24 \mathrm{c}$ \\
\hline
\end{tabular}

Means within a column having the same letters are not significantly differences according to Duncan's multiple range test (DMRT). 
Influence of foliar application of salicylic acid on growth and flowering ........

Table (10): Effect of diluted seawater irrigation, salicylic acid spray and their interaction on leaf $\mathrm{Na}, \mathrm{Cl}$ content, $\mathrm{Na} / \mathrm{K}$ ratio and survival \% of Calendula officinalis $\mathrm{L}$. during 2017/2018 and 2018/2019 seasons

\begin{tabular}{|c|c|c|c|c|c|c|c|c|c|}
\hline \multirow{2}{*}{\multicolumn{2}{|c|}{ Treatments }} & \multicolumn{2}{|c|}{$\mathrm{Na}(p p m)$} & \multicolumn{2}{|c|}{$\mathrm{Cl}(\mathrm{ppm})$} & \multicolumn{2}{|c|}{$\mathrm{Na} / \mathrm{K}$} & \multicolumn{2}{|c|}{ Survival \% } \\
\hline & & $\begin{array}{c}1 \underline{\text { st }} \\
\text { season }\end{array}$ & $\begin{array}{c}2 \frac{\text { nd }}{} \\
\text { season }\end{array}$ & $\begin{array}{c}11^{\text {st }} \\
\text { season }\end{array}$ & $\begin{array}{c}2^{\text {nd }} \\
\text { season }\end{array}$ & $\begin{array}{c}1 \underline{\text { st }} \\
\text { season }\end{array}$ & $\begin{array}{c}2^{\text {nd }} \\
\text { season }\end{array}$ & $\begin{array}{c}1 \underline{\text { st }} \\
\text { season }\end{array}$ & $\begin{array}{c}2^{\text {nd }} \\
\text { season }\end{array}$ \\
\hline \multicolumn{10}{|c|}{ Seawater (\%) } \\
\hline \multicolumn{2}{|c|}{ Cont. } & $0.765 d$ & $0.766 d$ & $0.231 d$ & $0.236 c$ & $0.486 d$ & $0.484 d$ & $100.00 a$ & $100.00 a$ \\
\hline \multicolumn{2}{|c|}{$10 \%$} & $0.805 \mathrm{c}$ & $0.806 c$ & $0.354 \mathrm{c}$ & $0.344 \mathrm{c}$ & $0.523 c$ & $0.520 c$ & $88.90 b$ & 87.44b \\
\hline \multicolumn{2}{|c|}{$20 \%$} & $0.825 b$ & $0.860 \mathrm{~b}$ & $0.843 b$ & $0.771 b$ & $0.555 b$ & $0.575 b$ & $83.50 \mathrm{c}$ & $82.62 \mathrm{c}$ \\
\hline \multicolumn{2}{|c|}{$30 \%$} & $0.888 a$ & $0.893 a$ & $0.918 a$ & $0.912 a$ & $0.615 a$ & $0.616 a$ & 75.38d & 74.04d \\
\hline \multicolumn{10}{|c|}{ Salicylic acid (ppm) } \\
\hline \multicolumn{2}{|l|}{0} & $1.013 a$ & $1.015 a$ & $0.682 a$ & $0.667 a$ & $0.690 a$ & $0.687 a$ & $87.54 a$ & $87.05 a$ \\
\hline \multicolumn{2}{|c|}{150} & $0.864 b$ & $0.866 \mathrm{~b}$ & $0.590 \mathrm{~b}$ & $0.592 a$ & $0.577 b$ & $574 b$ & $86.53 b$ & $85.00 a$ \\
\hline \multicolumn{2}{|c|}{300} & $0.586 c$ & $0.614 c$ & $0.488 c$ & $0.439 b$ & $0.368 c$ & $0.384 c$ & 86.77ab & $86.03 a$ \\
\hline Seawater & $\begin{array}{c}\text { SA } \\
(p p m)\end{array}$ & \multicolumn{8}{|c|}{ Interaction } \\
\hline \multirow{3}{*}{ Cont. } & 0 & $0.972 \mathrm{c}$ & $0.972 \mathrm{c}$ & $0.236 j$ & $0.237 e$ & $0.638 d$ & $0.634 d$ & $100.00 \mathrm{a}$ & $100.00 a$ \\
\hline & 150 & $0.784 \mathrm{~g}$ & $0.785 f$ & $0.234 j$ & $0.244 \mathrm{e}$ & $0.505 h$ & $0.503 \mathrm{~h}$ & $100.00 \mathrm{a}$ & $100.00 \mathrm{a}$ \\
\hline & 300 & $0.538 \mathrm{k}$ & $0.539 \mathrm{j}$ & $0.223 \mathrm{k}$ & $0.228 \mathrm{e}$ & $0.316 I$ & $0.315 I$ & $100.00 a$ & $100.00 \mathrm{a}$ \\
\hline \multirow{3}{*}{$10 \%$} & 0 & $0.982 b$ & $0.982 b$ & $0.431 \mathrm{~g}$ & $0.382 \mathrm{de}$ & $0.653 c$ & $0.649 c$ & $94.17 b$ & $93.33 b$ \\
\hline & 150 & $0.881 f$ & $0.882 e$ & $0.332 \mathrm{~h}$ & $0.332 \mathrm{de}$ & $0.589 \mathrm{~g}$ & $0.583 \mathrm{~g}$ & $86.17 \mathrm{c}$ & $84.33 \mathrm{c}$ \\
\hline & 300 & $0.553 \mathrm{j}$ & $0.555 i$ & $0.301 \mathrm{i}$ & $0.318 \mathrm{de}$ & $0.328 \mathrm{k}$ & $0.327 \mathrm{k}$ & $86.37 c$ & $84.67 \mathrm{c}$ \\
\hline \multirow{3}{*}{$20 \%$} & 0 & $0.984 b$ & $0.985 b$ & $0.986 b$ & $0.989 a b$ & $0.677 b$ & $0.674 b$ & $81.83 e$ & $81.53 \mathrm{e}$ \\
\hline & 150 & $0.890 \mathrm{e}$ & $0.895 d$ & $0.822 d$ & $0.817 b c$ & $0.600 f$ & $0.599 f$ & $83.67 d$ & 82.33de \\
\hline & 300 & $0.600 \mathrm{i}$ & $0.700 \mathrm{~g}$ & $0.722 \mathrm{e}$ & $0.506 d$ & $0.389 \mathrm{j}$ & $0.452 i$ & $85.00 \mathrm{~cd}$ & $84.00 \mathrm{~cd}$ \\
\hline \multirow{3}{*}{$30 \%$} & 0 & $1.113 a$ & $1.120 \mathrm{a}$ & $1.076 a$ & $1.059 a$ & $0.793 a$ & $0.792 a$ & $74.17 \mathrm{~g}$ & $73.33 \mathrm{~g}$ \\
\hline & 150 & $0.900 \mathrm{~d}$ & $0.900 \mathrm{~d}$ & $0.972 c$ & $0.975 a b$ & $0.613 e$ & $0.611 \mathrm{e}$ & $76.27 f$ & $73.33 \mathrm{~g}$ \\
\hline & 300 & $0.651 \mathrm{~h}$ & $0.661 \mathrm{~h}$ & $0.707 f$ & $0.703 c$ & $0.439 i$ & $0.441 \mathrm{j}$ & $75.70 f$ & $75.47 f$ \\
\hline
\end{tabular}

Means within a column having the same letters are not significantly differences according to Duncan's multiple range test (DMRT).

Foliar spraying of salicylic acid treatments $(150$ and $300 \mathrm{ppm})$ significantly increased leaf nitrogen, phosphorus, potassium, calcium $\%$, while sodium and chloride percentages as well as $\mathrm{Na} / \mathrm{K}$ ratio were decreased as compared with control treatment in both seasons. These results are in harmony with those of Hashish et al., (2015) and Abou El-Ftouh et al., (2018) on Calendula plants.
Regarding the interaction, there were significant differences among combination treatments in both seasons. The highest values of leaf $\mathrm{N}, \mathrm{P}, \mathrm{K}$ and $\mathrm{Ca}$ were obtained by the combination treatment of tap water (control) + high SA level, $300 \mathrm{ppm}$ in both seasons while the highest values as for $\mathrm{Na}, \mathrm{Cl}$ and $\mathrm{Na} / \mathrm{K}$ ratio were obtained with the combination treatment of diluted seawater irrigation at 20 and $30 \%+$ SA at 0 ppm (control). Furthermore, the highest significant 
values of survival \% were attained by the application of SA ( 0, 150 and 300) and control ( $0 \%$ seawater), also were obtained by SA foliar spraying at $\mathbf{1 5 0}$ and $\mathbf{3 0 0} \mathrm{ppm}$ with diluted seawater irrigation at $10 \%$ seawater in both seasons. These results agree with those reported by Bayat et al., (2012), and Pacheco et al., (2013).

\section{CONCLUSION}

It can be concluded that spraying calendula plant (Calendula officinalis $L$.) with salicylic acid at either 150 or 300 ppm when irrigated with diluted seawater had a positive effect on growth, flowering and increased the nutrition status and survival $\%$ of plants and also, it can be achievable to use diluted seawater at $\mathbf{1 0} \%$ seawater to irrigate it.

\section{REFERENCES}

Abou El-Ftouh, Z.A., M.A. Mohamed and A.K. Ibrahim (2018). Effect of saline water irrigation on growth and chemical composition of pot marigold plant. Arab Univ. J. Agric. Sci., Special Issue, 26(2A): 935-949.

Afzal, I., S.M.A. Basara, M. Faooq and A. Nawaz (2006). Alleviation of salinity stress in spring wheat by hormonal priming with $A B A$, salicylic acid and ascorbic acid. Int. J. Agric. Biol., 8:2328.

Arfan, M., R. Arthar and M. Ashraf (2007). Does exogenous application of salicylic acid through the rooting media modulate growth and photosynthetic capacity in two differently adopted durum wheat cultivars under salt stress. J. Plant Physiol, 164(6):685-694.

Ashraf, M. and P.J.C. Harris (2004). Potential biochemical indicators of salinity tolerance in plants. Plant Sci., 166: 3-16.

Baby, J., D. Jini and S. Sajatha (2010). Insight into the role of exogenous salicylic acid on plant grown under salt environment. Asian J of Crop Science, 2(4): 226-235.

Bafeel, S.O., K.G. Hanaa and Z.B. Alaa (2016). Effect of seawater irrigation on growth and some metabolites of jojoba plants. American-Eurasian J. Agric. and Environ. Sci., 16 (1): 49-59.

Basit, A., K. Shah, M. Rahman, L. Xing, X. Zuo, M. Han, N. Alam, F. Khan, I. Ahmed and M. A. Khalid (2018). Salicylic acid an emerging growth and flower inducing hormone in marigold (Tagetes sp. L.). Pure Appl. Biol., 7(4): 1301-1308.

Batanouny, K.H., M.M. Hussien and M.S. Abo El-Kheir (1988). Response of Zea mays to temporal variation of irrigation and salinity under form conditions in the Nile Delta Egypt, International Conferenceon on Plant Growth, Drought, and Salinity in Arab Region. Cairo Univ. Egypt.

Bates, L.S., R.P. Waldren and I.D. Teare (1973). Rapid determination of free proline for water stress studies. Plant and Soil, 39: 205-207.

Bayat, H., M. Alirezaie and H. Neamati (2012). Impact of exogenous salicylic acid on growth and ornamental characteristics of calendula (Calendula officianlis L.) under salinity stress. J. Stress Physiol. \& Biochem., 8(1), 258267.

Bricker, B. (1991). MSTATC: A Micro Computer Program from the Design Management and Analysis of Agronomic Research Experiments. Michigan State.

Brown, J.D. and O. Lilleland (1946). Rapid determination of potassium and sodium in plant material and soil extract by flame photometry. Proc. Amer. Soc. Hort. Sci., 48, 341-346.

Cassaniti, C., R. Daniela and T. J. Flowers (2012). The response of ornamental plants to saline irrigation water, 
irrigation - water management, pollution and alternative strategies, $\mathrm{Dr}$ Iker GarciaGarizabal (Ed.), ISBN: 978953-51-0421-6.

Chapman, H.D. and P.F. Pratt (1978). Methods of Analysis for Soils, Plant and Water. Univ. California USA.

Cheng, K.L. and R.H. Bray (1951). Determination of calcium and magnesium in soil and plants materials. Soil Sci., 72(6):449-458.

Delacerda, C.F., J. Cambraia and H.A. Ruiz (2003). Change in growth and in solute concentration in sorghum leaves and roots during salt stress recovery. Environ Exp. Bot.,54: 69-76.

Dole, J.M. and H.F. Wilkins (2004). Floriculture: Principles and Species. Prentice Hall, Upper Saddle River, New Jersey.

Duncan, B.D. (1955). Multiple range and multiple F-test. Biometrics, J. 11: 1-42.

El-Mahrouk, M.E., M.F. El-Nady and M.A. Hegazi (2010). Effect of diluted seawater irrigation and exogenous proline treatments on growth, chemical composition, and anatomical characteristics of Conocarpus erectus L. Journal Agriculture Research Kafrelsheikh University, 36(4): 420-446.

El- Sayed, N.I., W.M.F. Abd-ELhady and E. M. Selim (2017). Increased resistance to salt stress of Duranta plumieri irrigated with seawater by using thiamin, humic acid and salicylic acid. J. Plant Production, Mansoura Univ., 8 (5): 617 - 627.

Greenway, H. and R. Munns (1980). Mechanisms of salt tolerance in nonhalophytes. Annu. Rev. Plantphysiol., 31: 149-190.

Gul, H and R. Ahmad (2004). Effect of different irrigation intervals on growth of canola (Brassica napus L.) under different salinity levels. Pak J Bot., 36:359-372.
Hamburger, M., S. Adler, D. Baumann, A. Förg and B. Weinreich (2003). Preparative purification of the major anti-inflammatory triterpenoid esters from marigold (Calendula officinalis). Fitoterapia, 74(4):328338.

Hashish, K.H., A. Mazhar, S. Zaghloul, N. Abdel Aziz, M. Mahgoub and R. Eid (2015). Application of salicylic acid on Calendula officinalis $L$. is to alleviate the adverse effects of salinity stress. Intern. J. Chemtech Res., 8(6), 379-388.

Hayat, S.; B. Ali and A. Ahmad (2007). Salicylic Acid: Biosynthesis, Metabolism and Physiological Role in Blants. Salicylic acid: A plant Hormone. Springer Dordrecht 1-14.

Hussein, M.M., S.M. Shaaban and S. M. ElAshry (2010). Influence of diluted sea water and foliar fertilizer on hydro physical properties of a clayey soil in relation to growth, yield, and mineral status of millet. New York Science Journal; 3(7): 1-7.

Jackson, M.L. (1967). Soil Chemical and Plant Analysis. Prentice Hall of India, New Delhi.

Jin. M.G., R. Q. Zhang, L.F. Sun and Y.F. Gao (1999). Temporal and spatial soil water management: A case study in the Heilongjiang region, PR China. Agr. Water Manage, 42: 173 - 187.

Karlidage, H., E. Yildirim and A. Turan (2009). Exogenous application of salicylic acid affects quality and yield of strawberry grown under antifrost heated greenhouse conditions. J. Plant Nut. Soil Sci.,172: 270- 276.

Kraemer, E.O. and A.J. Stamm (1924). Mohr's method for the determination of silver and halogens in other than neutral solutions, J. Am. Chem. Soc., 46(12): 2707-2709.

Liu, Z.P., L. Liu., M.D. Chen., L. Q. Deng, G. M. Zhao, Q.Z. Tang and T.X. Xia (2003). Study on the irrigation systems in 
agriculture by seawater. J Nat Resour (in Chinese), 18: 423- 429.

Mazhar, A.A.M., A.E.d Rawya and N.G. Abd El. Aziz (2006). Effect of microbien under salt stress on nodulation, growth and chemical constituents of Sasbania aegyptiacain sandy soil. Bull. NRC. Egypt, 31: 247-268.

Munns, R., D.P. Schachtman and A.G. Condon (1995). The significance of a two-phase growth response to salinity in wheat and barley. Aust J Plant Physiol., 22: 561-569.

Murphy, J. and J.D. Riely (1962). A modified single solution method for the determination of phosphate in natural water. Anal. Chem. Acta, 27: 3136.

Nagata, M. and I. Yamashita (1992). Simple method for simultaneous determination of chlorophyll and carotenoids in tomato fruit. J. Japan. Soc. Food Sci. Technol., 39 (10): 925928.

Nofal, F.H., M.U. El-Segai and E.A. Seleem (2015). Response of Calendula officinalis L. plants to growth stimulants under salinity stress. American-Eurasian J. Agric. \& Environ. Sci., 15(9), 1767-1778.

Pacheco, A.C., C. Cabral, E. Fermino and C. Aleman (2013). Salicylic acidinduced changes to growth, flowering, and flavonoids production in marigold plants. J. of Med. Plants Res.,7(42): 3158-3163.

Raskin, I. (1992). Role of salicylic acid in plants. Annu Rev Plant Biol., 43: 439463.

Semiz, G.D and D. L. Suarez (2015). Tomato salt tolerance: impact of grafting and water composition on yield and ion relations. Turk $\mathrm{J}$ Agric For. 39:876-886.

Snedecor, G.W. and W.G. Cochran (1990). Statistical Methods. $7^{\text {th }}$ Ed. lowa State Univ. Press. Ames., lowa, USA. 593 pp.
Strobel, N. and J. Kuc (1995). Chemical and biological inducers of systemic resistance to pathogens protect cucumber and tobacco plants from damage caused by parquet and cupric chloride. Phytopathology (USA) No. 15044.

Turhan, A., H. Kuscu, N. Ozmen, M.S. Serbeci and A.O. Demir (2014). Effect of different concentrations of diluted seawater on yield and quality of lettuce. Chilean J. Agric. Res., 74(1): 111-116.

Van Breusegem, F., E. Vraneva and J. F. Dat (2001). The role of active oxygen species in plant signal transduction. Plant Sci., 161: 405-414.

Williams, M., T. Senaratna, K. Dixon and K. Sivasithamparam (2003). Benzoic acid induces tolerance to biotic stress caused by Phytophthora cinnamomi in Banksia attenuate. Plant Growth Regul, 41(1): 89-91.

Wood, C.W., D.W. Reeves and D.G. Himelrick (1993). Relationships between chlorophyll meter reading and leaf chlorophyll concentration, $\mathrm{N}$ status and crop yield. A review: Proc. Agro. Soc. N.Z., 23: 1-9.

Xiao-Hua, L., C. Jin-He, L. Ling, L. Qing and L. Zhao-Pu (2009). Effect of seawater stress on physiological and biochemical responses of five Jerusalem artichoke ecotypes. Soil Sci. Soc. of China, 19: 208-216.

Yao, X., R. Yang, F. Zhao, S. Wang, C. Li and $W$. Zhao (2016). An analysis of physiological index of differences in drought tolerance of tomato rootstock seedlings. J Plant Biol., 59:311-321.

Yusuf, M., S. Hayat, M. Alyemeni, Q. Fariduddin and A. Ahmad (2013). Salicylic acid: physiological roles in plants. Chapter: efficiency of salicylic acid application on postharvest perishable crops, Springer Netherland, (p: 15-30). 


\title{
تأثير الرش بحمض السالسيليك على نمو وإهار نبات الاقحوان تحت مستويات مختلفة من ملوحة مياه الري
}

\author{
محمد السيد عبد الجيد
}

قسم بحوث الحائق النباتية -معهد بحوث البساتين - مركز البحوث الزراعية-الجيزة - مصر

الملخص العربي

التغيرات المناخية أدت الى التقليل من مصادر المياه العذبة مما أدى الى زيادة الحاجة لمياه الرى. ولهذا السبب كان

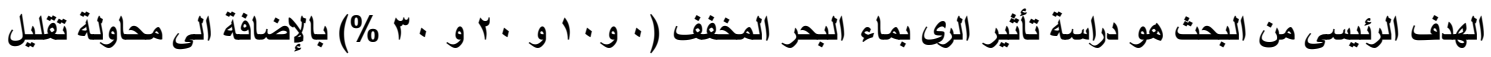

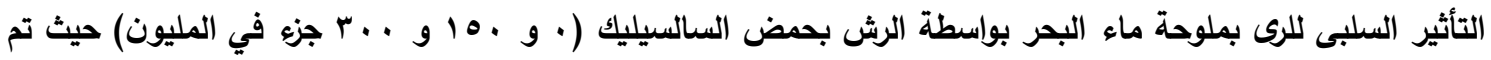

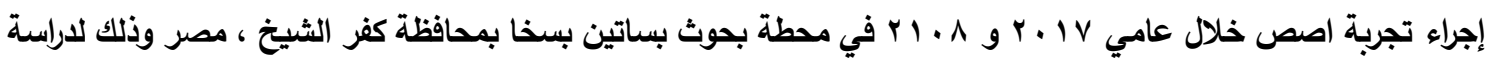
تأثثر الرى بماء البحر المخفف والرش بحمض السلسيلك والتفاعل بينهما على النمو والازهار ونسبة البقاء ، وكذلك التركيب

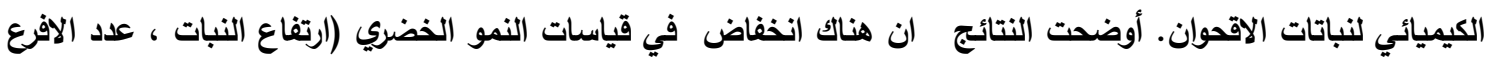

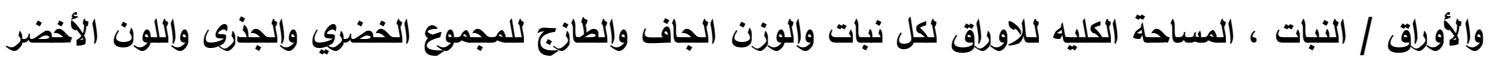

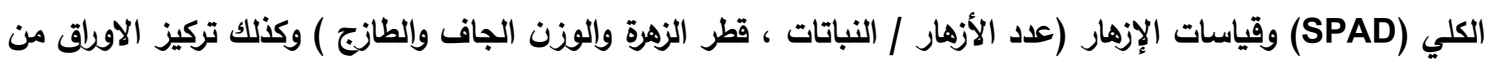

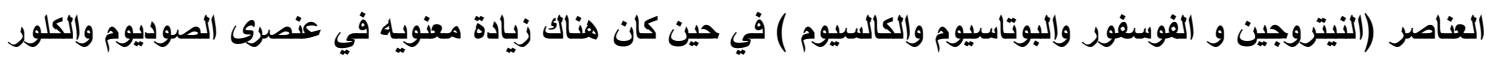

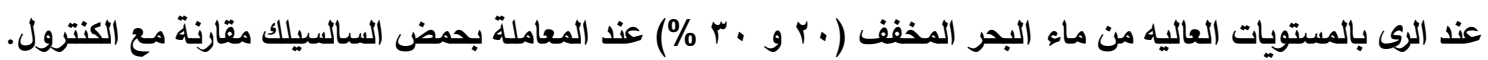

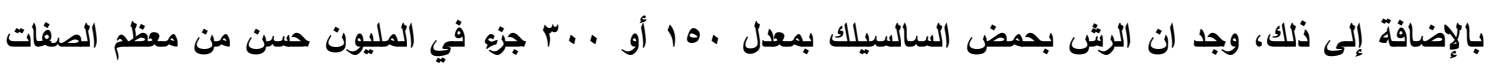
السابقة تحت ظروف الرى بالماء المالح.

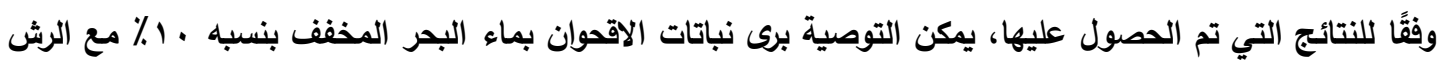
بحمض السالسيلك بمعل . . ج جزء في المليون حيث سجلت أفضل النتائج من حيث النمو ، والازهار ونسبة البقاء. 
